Article

\title{
Sensitivity Enhancement in Si Nanophotonic Waveguides Used for Refractive Index Sensing
}

\author{
Yaocheng Shi *, Ke Ma and Daoxin Dai \\ Centre for Optical and Electromagnetic Research, State Key Laboratory for Modern Optical Instrumentation, \\ Zhejiang Provincial Key Laboratory for Sensing Technologies, Zhejiang University, Zijingang Campus, \\ Hangzhou 310058, China; make@coer-zju.org (K.M.); dxdai@zju.edu.cn (D.D.) \\ * Correspondence: yaocheng@zju.edu.cn; Tel.: +86-571-8820-6516
}

Academic Editor: Vittorio M. N. Passaro

Received: 6 January 2016; Accepted: 29 February 2016; Published: 3 March 2016

\begin{abstract}
A comparative study is given for the sensitivity of several typical Si nanophotonic waveguides, including SOI (silicon-on-insulator) nanowires, nanoslot waveguides, suspended Si nanowires, and nanofibers. The cases for gas sensing $\left(n_{\mathrm{cl}} \sim 1.0\right)$ and liquid sensing $\left(n_{\mathrm{cl}} \sim 1.33\right)$ are considered. When using SOI nanowires (with a $\mathrm{SiO}_{2}$ buffer layer), the sensitivity for liquid sensing $(S \sim 0.55)$ is higher than that for gas sensing $(S \sim 0.35)$ due to lower asymmetry in the vertical direction. By using SOI nanoslot waveguides, suspended Si nanowires, and Si nanofibers, one could achieve a higher sensitivity compared to sensing with a free-space beam $(S=1.0)$. The sensitivity for gas sensing is higher than that for liquid sensing due to the higher index-contrast. The waveguide sensitivity of an optimized suspended Si nanowire for gas sensing is as high as 1.5, which is much higher than that of a SOI nanoslot waveguide. Furthermore, the optimal design has very large tolerance to the core width variation due to the fabrication error $(\Delta w \sim \pm 50 \mathrm{~nm})$. In contrast, a Si nanofiber could also give a very high sensitivity (e.g., $~ 1.43$ ) while the fabrication tolerance is very small (i.e., $\Delta w< \pm 5 \mathrm{~nm}$ ). The comparative study shows that suspended $\mathrm{Si}$ nanowire is a good choice to achieve ultra-high waveguide sensitivity.
\end{abstract}

Keywords: sensitivity; silicon; nanowire; nanoslot; nanofiber

\section{Introduction}

Optical waveguide sensors are paving the way for realizing low-cost, highly sensitive, ultra-compact optical sensors, which are desired for many applications such as biological, environmental and chemical detections [1-9]. It is also easy to have a sensor array based on optical waveguides. Usually, the principle of optical waveguide sensors is based on the perturbation of the field of a guided mode caused by optical absorptions, fluorescence or refractive index changes of the measured sample [8]. Among them, refractive index sensors are popular because of their easy realization, and the potential for real-time monitoring with a minimal sample volume. When the concentration of the sample covering on the waveguide surface changes, the effective refractive index of the optical waveguide changes and consequently a phase shift will be introduced. This phase shift could be converted into an intensity change or a frequency shift using interferometers or resonant structures. In past years, people have developed various integrated optical sensors based on different structures and mechanisms, e.g., Mach-Zehnder interferometers (MZI) [1], and high-Q optical microcavities (including microrings/microdisks [2-11]).

When designing and fabricating an optical sensor, sensitivity is one of the most important figures of merit to consider. Generally speaking, there are two parts contributing to the total sensitivity, i.e., the waveguide sensitivity $\left(\mathrm{S}_{\mathrm{WG}}\right)$ and the device sensitivity $\left(\mathrm{S}_{\mathrm{d}}\right)$ [11]. The device sensitivity is the ratio of the change in the measured optical parameter (i.e., the resonance wavelength, or the intensity at a 
specific wavelength) to the change of the effective index. The waveguide sensitivity $\mathrm{S}_{\mathrm{WG}}$ is defined as the ratio of the effective index change $\Delta \mathrm{n}_{\text {eff }}$ to the change $\Delta \mathrm{n}_{\mathrm{s}}$ of the sample index, i.e., $\mathrm{S}_{\mathrm{WG}}=\Delta \mathrm{n}_{\mathrm{eff}} / \Delta \mathrm{n}_{\mathrm{s}}$. In order to improve the sensitivity of an optical sensor, one should improve the device design as well as the waveguide design. The device sensitivity $S_{d}$ mainly depends on the device structure while the waveguide sensitivity $S_{W G}$ depends on the waveguide cross section as well as the refractive index profile. One can optimize the device structure and the waveguide structure separately to maximize the device sensitivity and the waveguide sensitivity, respectively. For example, an ultra-high sensitivity was achieved using the Vernier effect in a dual-ring system [12-14], and the waveguide sensitivity can be improved using some special waveguides, which will be discussed in this paper.

Since only the evanescent field (which is a small part of the total guided-modal field) "experiences" the analyzed medium, the sensitivity SWG of a guided mode in an optical waveguide is usually assumed to be smaller or much smaller than that of a free-space beam $(S=1)$ [15]. This is true when using a conventional strip or rib waveguide with a large core size and a low index-contrast because the evanescent fields are not very strong. For example, the sensitivity for a $\mathrm{SiO}_{2}$ or polymer waveguide is usually less than 0.1 [1], which is much smaller than that of a free-space beam. Hollow-core waveguides [16] are developed to obtain a higher sensitivity in the way of guiding light in the low-index sample material using the Bragg-grating effect. However, this also makes the waveguide transmission highly wavelength dependent and furthermore the fabrication is quite complicated.

Recently, Si nanowires have become a favored choice because of their evanescent field enhancement in the cladding region due to the small cross section and the ultra-high index contrast. TM polarization is usually used to have higher sensitivity, $\mathrm{S}_{\mathrm{WG}} \approx 0.5$ [1], which, however, is still less than that of a free-space beam ( $\mathrm{S}=1)$. In 2004, a nanoslot waveguide was introduced as a novel guided-wave configuration [17], in which there is a field enhancement in the low-index slot region due to the boundary condition of the perpendicular electrical component. This makes it very attractive to achieve high sensitivity for optical sensing [18-20]. For a nanoslot waveguide, the optimized waveguide sensitivity could be as high as $1.0[19,20]$, which provides a way to realizing a waveguide sensitivity of more than the sensitivity of a free-space beam $(S=1)$. In [15], the author gave an analytical analysis for a three-layer slab waveguide and reported a peculiar effect, namely that the waveguide sensitivity can indeed be larger than 1.0 for TM polarization. This means that an optical waveguide sensor can be made with a higher sensitivity than a free-space configuration.

In this paper, we consider the case with three-dimensional nanophotonic waveguides and give a comparative study for the sensitivity of several typical silicon nanophotonic waveguides with a very-high index-contrast, e.g., SOI (silicon-on-insulator) nanowires, SOI nanoslot waveguides, suspended Si nanowires, and Si nanofibers. Our simulation shows that an enhanced sensitivity of about 1.5 could be achieved by using suspended Si nanowires.

\section{Analysis and Discussion}

In this paper, we consider several typical Si nanophotonic waveguides operating at $1550 \mathrm{~nm}$. The involved materials for nanophotonic waveguides include $\mathrm{Si}, \mathrm{SiO}_{2}$, and gas (or liquid), whose refractive indices are assumed to be 3.455, 1.445, and 1.0 (or 1.33), respectively. In this paper, we assume that the buffer layer in various SOI optical waveguides considered here is thick enough to make the substrate leakage negligible. For example, the thickness of the $\mathrm{SiO}_{2}$ buffer layer is $3 \mu \mathrm{m}$ in the following calculation.

\subsection{SOI Nanowires}

In this part, we consider a $\mathrm{SOI}$ nanowire, which has a $\mathrm{Si}$ core and a $\mathrm{SiO}_{2}$ buffer layer, as shown in Figure 1a, which is the most popular one used for optical sensing [1]. The upper-cladding is the sample to be measured (i.e., $\left.\mathrm{n}_{\mathrm{s}}=\mathrm{n}_{\mathrm{cl}}\right)$, which is a gas $\left(\mathrm{n}_{\mathrm{s}}=\mathrm{n}_{\mathrm{cl}} \sim 1\right)$ or liquid $\left(\mathrm{n}_{\mathrm{s}}=\mathrm{n}_{\mathrm{cl}} \sim 1.33\right)$. TM polarization is considered in the following calculation. Figure $1 \mathrm{~b}$ shows the calculated field distribution of the TM 
fundamental mode for a Silicon nanowire waveguide with $\mathrm{h}_{\mathrm{co}}=250 \mathrm{~nm}, \mathrm{w}_{\mathrm{co}}=350 \mathrm{~nm}, \mathrm{n}_{\mathrm{cl}}=1.0$ as an example.

Figure 1c shows the calculated sensitivity as the core width $\mathrm{w}_{\mathrm{co}}$ varies from $0.1 \mu \mathrm{m}$ to $0.8 \mu \mathrm{m}$ when the upper-cladding is filled with gas $(\mathrm{n} \sim 1.0)$. The Si core layer is 220, 230, 240, 250, 260, 270, 280, 290, 300, 350, and $400 \mathrm{~nm}$ thick, respectively. For the case with a relatively thick core (e.g., $\mathrm{h}_{\mathrm{co}} \geqslant 350 \mathrm{~nm}$ ), the sensitivity is low, especially when the core width is large. This is because most power of the fundamental mode is confined in the core region while the evanescent field is very small, as shown in Figure $1 \mathrm{~d}$, where $\mathrm{P}_{\mathrm{co}}, \mathrm{P}_{\mathrm{cl}}$, and $\mathrm{P}_{\text {buffer }}$ are the power confinement ratio in the regions of core, upper-cladding, and buffer, respectively. When the core width decreases, the confinement becomes weaker and more evanescent field penetrates to the upper-cladding (see Figure 1d). The sensitivity consequently increases, as shown in Figure 1c.

On the other hand, one should note that the present waveguide is asymmetrical in the vertical direction, i.e., the upper-cladding (gas) has a lower refractive index than the buffer layer $\left(\mathrm{SiO}_{2}\right)$. Therefore, when the core becomes very narrow, the optical waveguide cannot confine the optical field well and less power is confined in the upper-cladding region, while more power moves to the $\mathrm{SiO}_{2}$ buffer region (see Figure 1d). For example, when $\mathrm{w}_{\mathrm{co}}=140 \mathrm{~nm}$, the power ratios in the regions of $\mathrm{Si}$ core, $\mathrm{SiO}_{2}$ buffer, and gas upper-cladding are $25.4 \%, 41.6 \%$, and $32.9 \%$, respectively. Thus, the sensitivity becomes smaller as the core width decreases further. This explains the existence of an optimal core width $w_{\text {opt }}$ for a maximal sensitivity when the core width varies in Figure 1c. For example, when $\mathrm{h}_{\mathrm{co}}=400 \mathrm{~nm}$, the maximal sensitivity $\mathrm{S}_{\max }=0.441$ when choosing the optimal core width $\mathrm{w}_{\mathrm{opt}}=130 \mathrm{~nm}$. However, the sensitivity decreases significantly when the core width deviates from the optimal value. Furthermore, such a SOI nanowire with a high aspect ratio is not a good option when considering to the fabrication (e.g., the etching process) and the scattering loss. When choosing a thinner SOI nanowire, there is also an optimal core width $\mathrm{w}_{\text {opt }}$ for maximal sensitivity. A significant decrease of the sensitivity is also observed when choosing a core width much smaller than the optimal width $w_{\text {opt }}$. And the sensitivity around $w=w_{\text {opt }}$ is less dependent on the core width in comparison to the case with a large thickness (e.g., $\mathrm{h}_{\mathrm{co}}=400 \mathrm{~nm}$ ). Therefore, one could achieve high sensitivity when the core width varies in a large range around $\mathrm{w}_{\mathrm{opt}}$.

From Figure 1c, we also observe that the sensitivity curve for thin SOI nanowires is discontinuous as the core width decreases from $0.8 \mu \mathrm{m}$ to $0.6 \mu \mathrm{m}$. This is due to the mode hybridization in that width range. In order to explain this, Figure $1 \mathrm{~d}$ shows the effective index of an SOI nanowire with $\mathrm{h}_{\mathrm{co}}=250 \mathrm{~nm}$ as the core width varies. From this figure, it can be seen that there is a region (see the circle in Figure 1e) around $\mathrm{w}_{\mathrm{co}}=700 \mathrm{~nm}$, where the TM fundamental mode $\left(\mathrm{TM}_{0}\right)$ and the first-order mode of TE polarization ( $\left.\mathrm{TE}_{1}\right)$ are hybridized. Therefore, when we consider the sensitivity of $\mathrm{TM}_{0}$, a jump appears, as shown in Figure 1c. As can be seen, higher sensitivity $S_{W G}$ can be achieved when choosing the waveguide width around the mode hybridization region. However, when higher-order modes are involved, there might be some undesired multimode effect. For example, when using a microring-resonator sensor, more resonance peaks will appear and the $\mathrm{Q}$-factor will degrade. Therefore, we still focus on the singlemode silicon optical waveguides for optical sensing. Here we also calculate the sensitivity for SOI nanowires with a liquid cladding $(\mathrm{n} \sim 1.33)$, which is very popular for biosensing. Figure 1f shows the calculated sensitivity, which is similar to the case for the gas sensing (see Figure 1c). When there is a liquid cladding, the optical waveguide becomes less asymmetrical because the liquid index is closer to that of the $\mathrm{SiO}_{2}$ buffer layer. Therefore, less power is confined in the $\mathrm{SiO}_{2}$ buffer region and more power is confined in the upper-cladding region, as shown in Figure 1g. Thus, more evanescent optical fields interact with the sample and consequently the sensitivity of a SOI nanowire becomes higher for liquid sensing than that for gas sensing (see Figure 1c,f). 


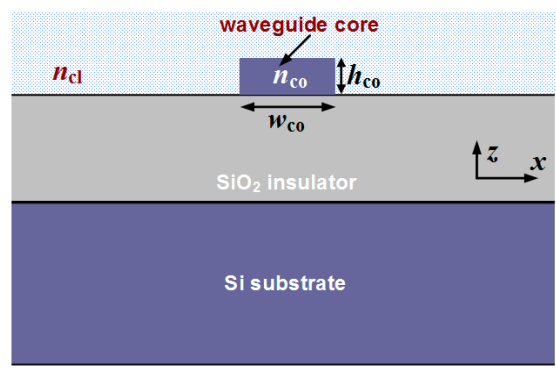

(a)

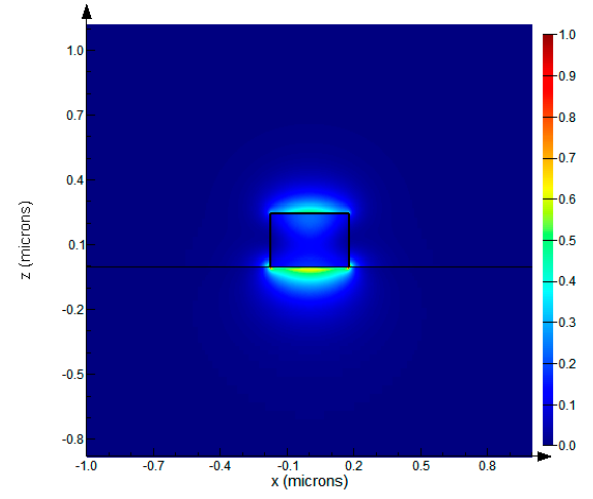

(b)

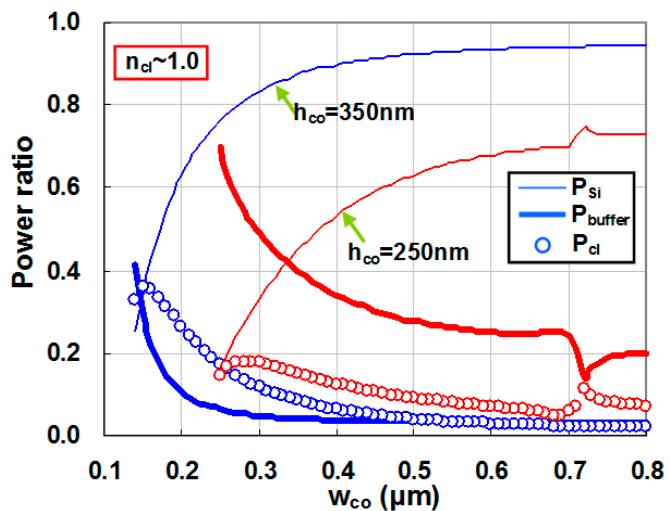

(d)

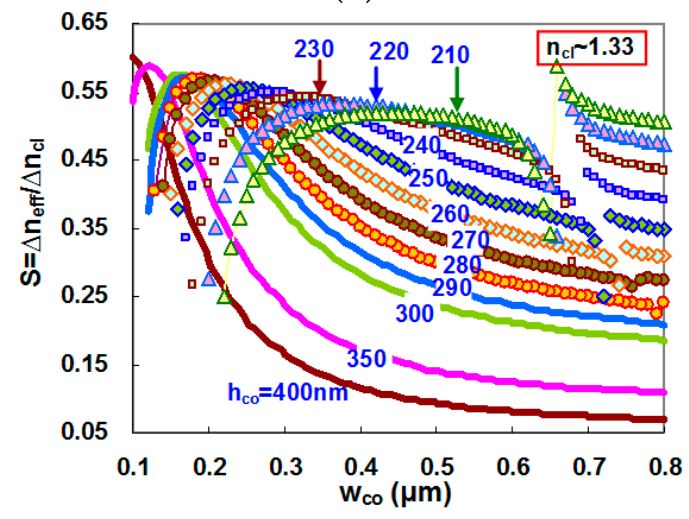

(f)

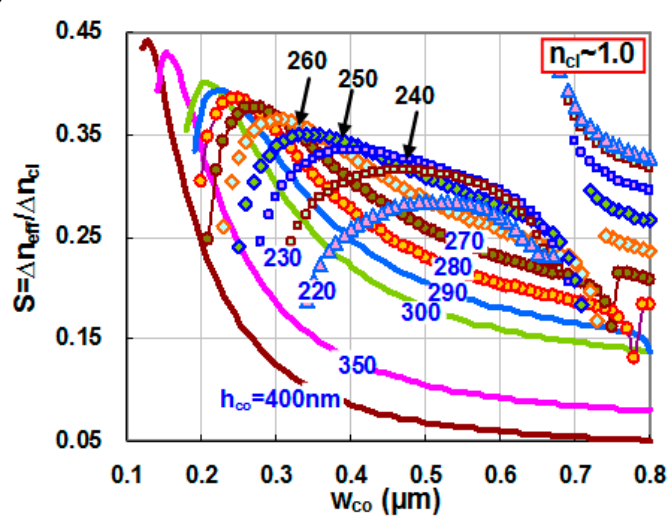

(c)

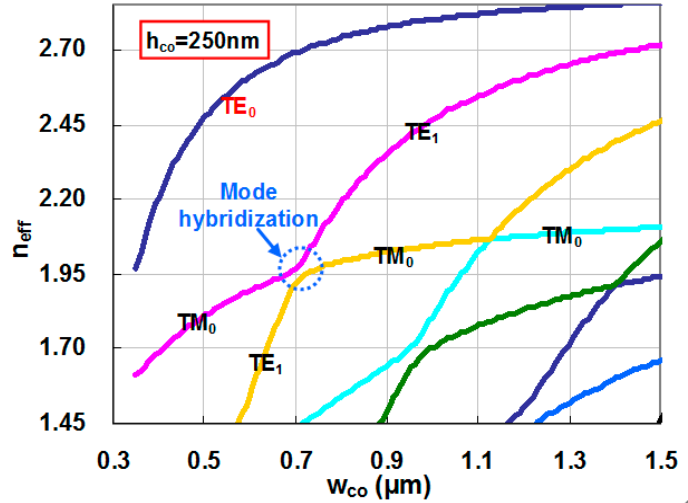

(e)

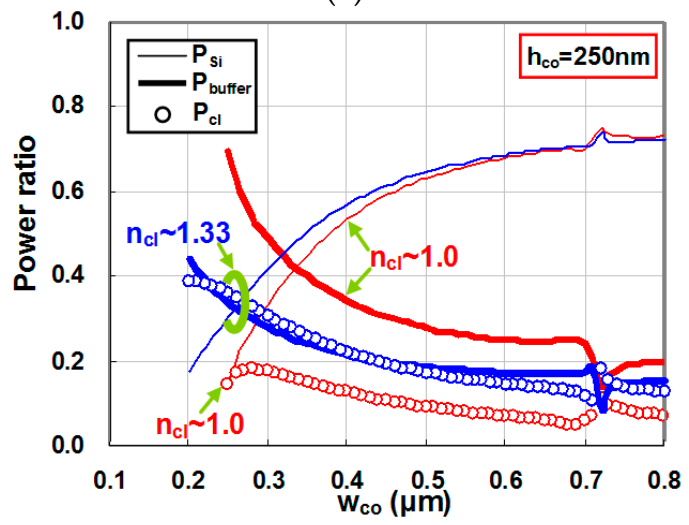

(g)

Figure 1. (a) The cross section of a SOI nanowire; (b) the calculated field distribution of the TM fundamental mode for a Silicon nanowire waveguide with $\mathrm{h}_{\mathrm{co}}=250 \mathrm{~nm}, \mathrm{w}_{\mathrm{co}}=350 \mathrm{~nm}$, and $\mathrm{n}_{\mathrm{cl}}=1.0$; (c) the calculated waveguide sensitivity as the core width varies when the upper cladding is filled with gas $(\mathrm{n} \sim 1.0)$; $(\mathrm{d})$ the power confinement ratio in the regions of core $\left(\mathrm{P}_{\mathrm{co}}\right)$, upper-cladding $\left(\mathrm{P}_{\mathrm{cl}}\right)$, and buffer $\left(\mathrm{P}_{\text {buffer }}\right)$; (e) the effective indices of the eigenmodes for a SOI nanowire with $\mathrm{h}_{\mathrm{co}}=250 \mathrm{~nm}$; (f) the calculated sensitivity as the core width varies when the upper cladding is filled with liquid ( $\mathrm{n} \sim 1.33$ ); and $(\mathrm{g})$ the power ratio in the regions of core $\left(\mathrm{P}_{\mathrm{co}}\right)$, upper-cladding $\left(\mathrm{P}_{\mathrm{cl}}\right)$, and buffer (Pbuffer) when $\mathrm{h}_{\mathrm{co}}=250 \mathrm{~nm}$. 


\subsection{SOI Nanoslot Waveguides}

An SOI nanoslot waveguide consists of two Si regions with a nanoslot between them, as shown in Figure 2a. Figure $2 \mathrm{~b}$ shows the calculated field distribution of the TE fundamental mode for $\mathrm{a}$ SOI nanoslot waveguide with $\mathrm{h}_{\mathrm{co}}=400 \mathrm{~nm}, \mathrm{w}_{\mathrm{co}}=180 \mathrm{~nm}$, and $\mathrm{w}_{\mathrm{s}}=30 \mathrm{~nm}$ as an example. It can be seen that there is a significant field enhancement in the nanoslot region. This field enhancement makes it very attractive for optical sensing when the sample to be measured fills the whole region of upper-cladding (including the nanoslot region).

Figure 2c-f show the calculated waveguide sensitivity for gas sensing as well as liquid sensing as the core width $\mathrm{w}_{\mathrm{co}}$ varies for the cases of $\mathrm{h}_{\mathrm{co}}=400,350,300$, and $250 \mathrm{~nm}$, respectively.The nanoslot width is chosen as 100, 80, 60, 50, 40, and $30 \mathrm{~nm}$ for any given core height $\mathrm{h}_{\mathrm{co}}$. In Figure $2 \mathrm{c}-\mathrm{f}$, it can be seen that the sensitivity of a nanoslot waveguide is similar for different values of $h_{c o}$ when it is covered by gas or liquid. For a nanoslot waveguide with a given slot width $\mathrm{w}_{\mathrm{s}}$ and core height $\mathrm{h}_{\mathrm{co}}$, the sensitivity increases rapidly and then decreases slightly as the core width varies in the range from $0.12 \mu \mathrm{m}$ to $0.24 \mu \mathrm{m}$. It can be seen that there exists an optimal core width $\mathrm{w}_{\text {opt }}$ for obtaining a maximal sensitivity $S_{\max }$ for both cases based on claddings of gas and liquids.

When the core width becomes small (e.g., $\mathrm{w}_{\mathrm{co}}=0.1 \mu \mathrm{m}$ ), the optical confinement is very weak and the power at the under-cladding becomes dominant due to the vertical asymmetry, especially in the case with a gas cladding. In this case, the optical waveguide become insensitive to the index change of the upper-cladding. In contrast, when there is a liquid cladding, the vertical asymmetry is alleviated and consequently the sensitivity is still relatively high even when the $\mathrm{Si}$ core width is reduced to $0.1 \mu \mathrm{m}$. On the other hand, when the core width becomes larger (e.g., $0.24 \mu \mathrm{m}$ ), the optical confinement becomes stronger and more power is confined in the Si core region, and consequently the field enhancement in the nanoslot is significantly limited. Therefore, the sensitivity of the nanoslot optical waveguide decreases as the core width increases.

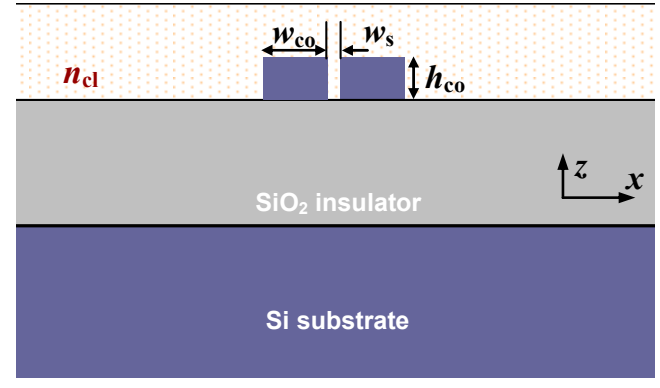

(a)

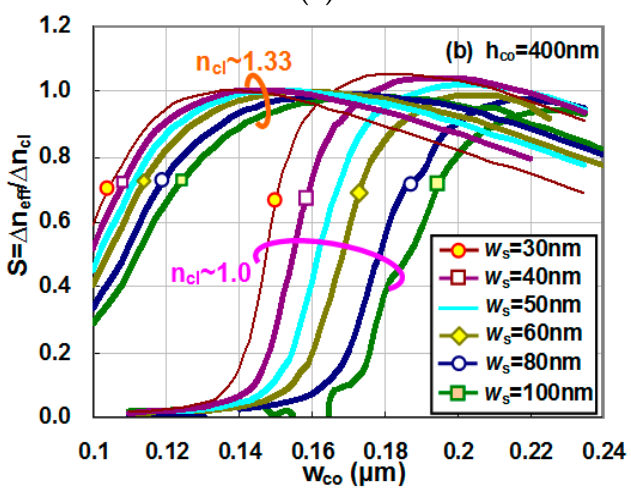

(c)

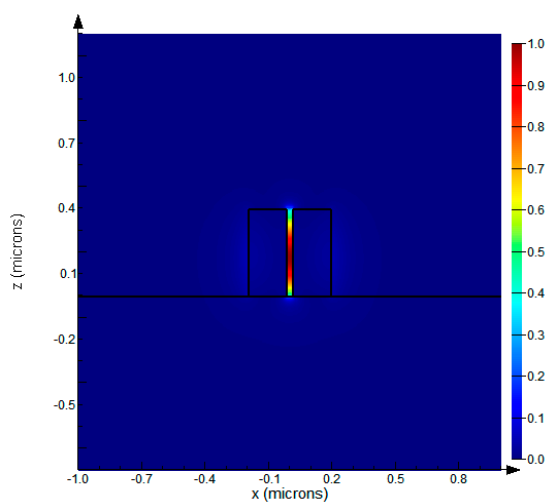

(b)

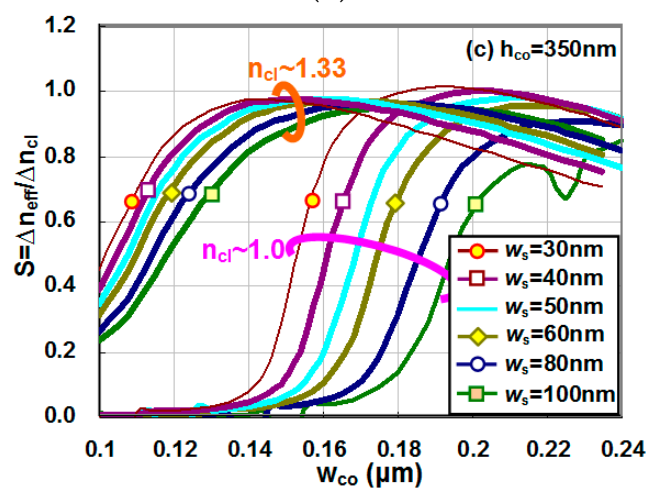

(d)

Figure 2. Cont. 


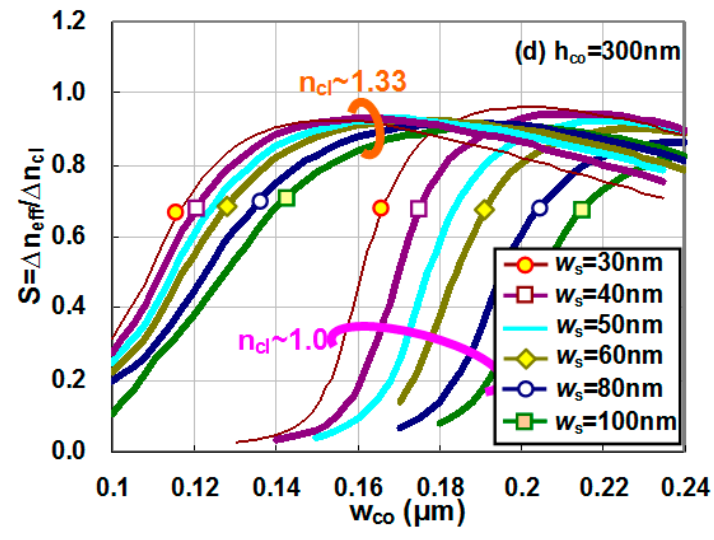

(e)

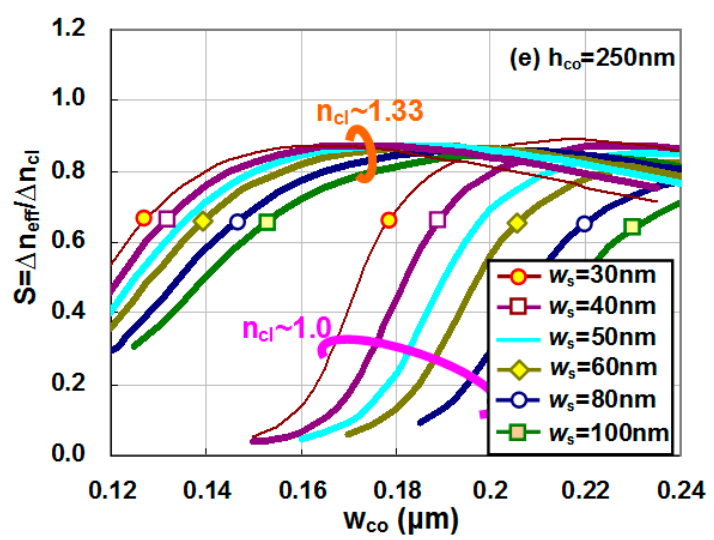

(f)

Figure 2. (a) The cross section of a nanoslot optical waveguide; and (b) the calculated field distribution of the TE fundamental mode for a SOI nanoslot waveguide with $\mathrm{h}_{\mathrm{co}}=400 \mathrm{~nm}, \mathrm{w}_{\mathrm{co}}=180 \mathrm{~nm}$, and $\mathrm{w}_{\mathrm{S}}=30 \mathrm{~nm}$ as an example. The calculated sensitivity for gas sensing and liquid sensing as the core width varies for the cases with different core heights: (c) $h_{c o}=400 \mathrm{~nm} ;(d) h_{c o}=350 \mathrm{~nm} ;(e) h_{c o}=300 \mathrm{~nm}$; and (f) $h_{\mathrm{co}}=250 \mathrm{~nm}$.

We also note that a nanoslot waveguide with a larger core height gives a higher maximal sensitivity, which could be larger than that of a free-space beam $(S=1)$. This is because a larger optical field overlaps with the upper-cladding when the core height increases. In contrast, when the core height turns small, more power moves down to the $\mathrm{SiO}_{2}$ under-cladding because of the asymmetry in the vertical direction. From Figure $2 \mathrm{c}-\mathrm{f}$ and Figure $1 \mathrm{~b}$, it can be seen that nanoslot waveguides have a much higher sensitivity than conventional SOI nanowires.

\subsection{Suspended Si Nanowires}

In this part, we give an analysis for a suspended Si nanowire, which includes a Si core surrounded by the sample, as shown in Figure 3a. Such a suspended waveguide could be achieved with supported arms [21,22]. Figure $3 b$ shows the calculated field distribution of the TM fundamental mode for a suspended Si nanowire waveguide with $\mathrm{h}_{\mathrm{co}}=220 \mathrm{~nm}, \mathrm{w}_{\mathrm{co}}=525 \mathrm{~nm}$, and $\mathrm{n}_{\mathrm{cl}}=1.0$ as an example. Figure $3 \mathrm{c}$ shows the calculated sensitivity for a suspended Si nanowire with different core heights $h_{c o}$ as the core width varies from $0.1 \mu \mathrm{m}$ to $0.8 \mu \mathrm{m}$. From this figure, it can be seen that the sensitivity is slightly higher than 1.0 (i.e., the sensitivity of free space) when the core height is small (e.g., $150 \mathrm{~nm}$ ). This is because the power confined in the Si core is very small while most power of the guided mode locates at the surrounded media, which is very similar to the free space case.

When the core height becomes larger, a significant sensitivity enhancement is observed. For a suspended Si nanowire with a fixed core height (e.g., $\mathrm{h}_{\mathrm{co}}=100 \sim 200 \mathrm{~nm}$ ), the sensitivity increases monotonously when the core width ranges from $0.1 \mu \mathrm{m}$ to $0.8 \mu \mathrm{m}$. When increasing the core height further (e.g., $\mathrm{h}_{\mathrm{co}}=250,240,230,220$, and $210 \mathrm{~nm}$ ), one sees that in the range of $0.1 \mu \mathrm{m}<\mathrm{w}_{\mathrm{co}}<0.8 \mu \mathrm{m}$ there exists an optimal core width $\mathrm{w}_{\text {co_opt }}$ (which gives a maximal sensitivity $S_{\max }$ of $>1.0$ ).

In order to explain this, one can use the formula for the sensitivity of a three-layer slab optical waveguide given in [15], i.e.,

$$
S=\frac{n_{\mathrm{eff}}}{n_{\mathrm{cl}}}\left(2-\frac{n_{\mathrm{cl}}^{2}}{n_{\mathrm{eff}}^{2}}\right) \frac{P_{\mathrm{cl}}}{P_{\mathrm{tot}}}
$$

where $\mathrm{n}_{\mathrm{eff}}$ is the effective index, $\mathrm{n}_{\mathrm{cl}}$ is the index of the cladding, and $\mathrm{P}_{\mathrm{cl}} / \mathrm{P}_{\text {tot }}$ is the power confinement ratio in the cladding region. In Figure $3 d$, we show the power confinement ratio in the regions of core and cladding. In this figure, one sees that the power confinement ratio in the cladding region increases monotonously as the core width decreases, which is easy to understand. Intuitively speaking, one expects to have a higher sensitivity when more power is confined in the cladding region. However, 
according to Equation (1), the sensitivity depends not only on the ratio of $\mathrm{P}_{\mathrm{cl}} / \mathrm{P}_{\text {tot }}$ but also the ratio of $\mathrm{n}_{\mathrm{eff}} / \mathrm{n}_{\mathrm{cl}}$. When the core width decreases, the ratio $\mathrm{P}_{\mathrm{cl}} / \mathrm{P}_{\text {tot }}$ increases while the ratio $\mathrm{n}_{\mathrm{eff}} / \mathrm{n}_{\mathrm{cl}}$ decreases, which causes an optimal core width for obtaining a maximal sensitivity.

One can imagine that a wide suspended waveguide should have similar result with a slab waveguide. Figure 3e gives a comparison between the calculated sensitivities for a suspended Si nanowire with $\mathrm{w}_{\mathrm{CO}}=0.8 \mu \mathrm{m}$ and $\mathrm{w}_{\mathrm{CO}}=\infty$ (slab waveguide). Here, the results for the slab waveguide $\left(w_{\mathrm{co}}=\infty\right)$ is calculated with Equation (1). It can be seen that these results are quite similar and there is an optimal core height $h_{\text {co }}$ around $h_{c o}=220 \mathrm{~nm}$ for the maximal sensitivity. For the suspended waveguide with a given core height $h_{\mathrm{co}}$, Figure $3 \mathrm{c}$ shows that the optimal core width for the maximal sensitivities $S_{\max }$ are slightly dependent on the core height. One should choose $h_{\text {co }}=220 \mathrm{~nm}$ to maximize the sensitivity and the corresponding maximal sensitivity $S_{\max }$ is close to 1.5 , which is much higher than 1.0 (which is the sensitivity in a free space system). This high sensitivity is due to the high index contrast between the core and the surrounding media (see Equation (1)). The design of $\mathrm{h}_{\mathrm{co}}=220 \mathrm{~nm}$ also has a large tolerance to the variation of the core width. Even when the core width deviates from its optimal value $\mathrm{w}_{\mathrm{co} \_ \text {opt }}(=0.525 \mu \mathrm{m})$, the sensitivity does not decrease much. In comparison with the sensitivity for a SOI nanoslot waveguide (shown in Figure 2c-f), the suspended Si nanowire could provide a much higher sensitivity even though it does not have a field enhancement in the low-index region. Such a significant sensitivity enhancement in a suspended Si nanowire makes it very attractive for the application of optical sensing, especially since it is also relatively easy to fabricate.

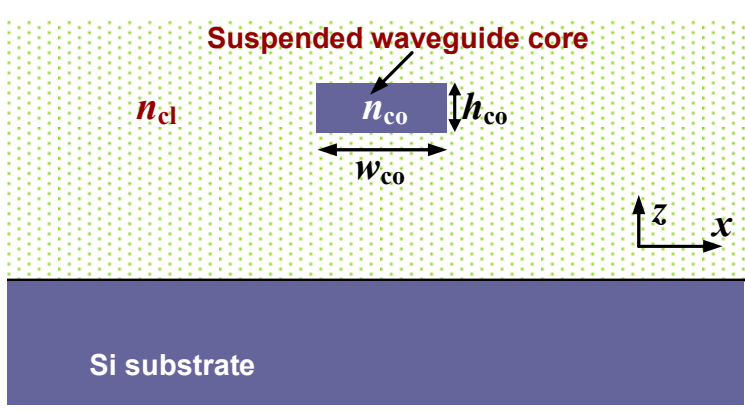

(a)

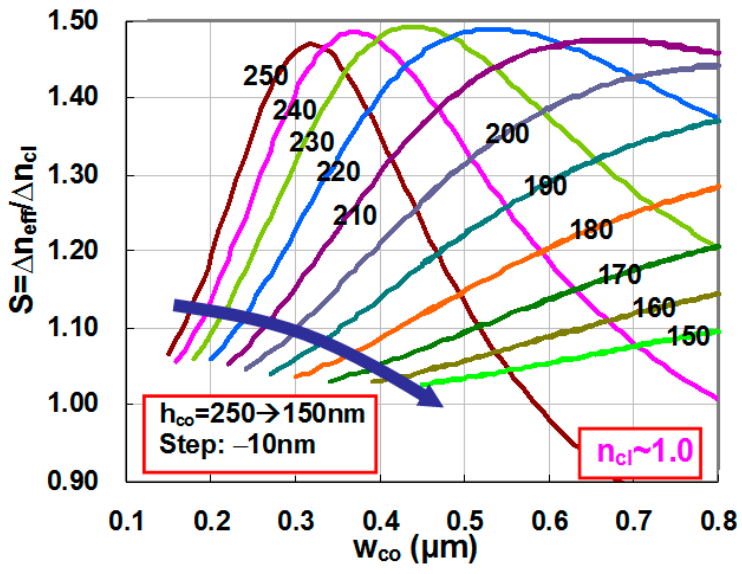

(c)

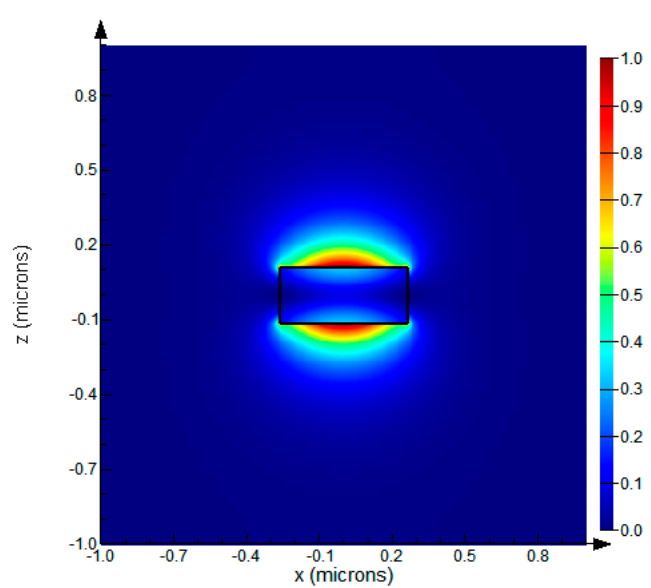

(b)

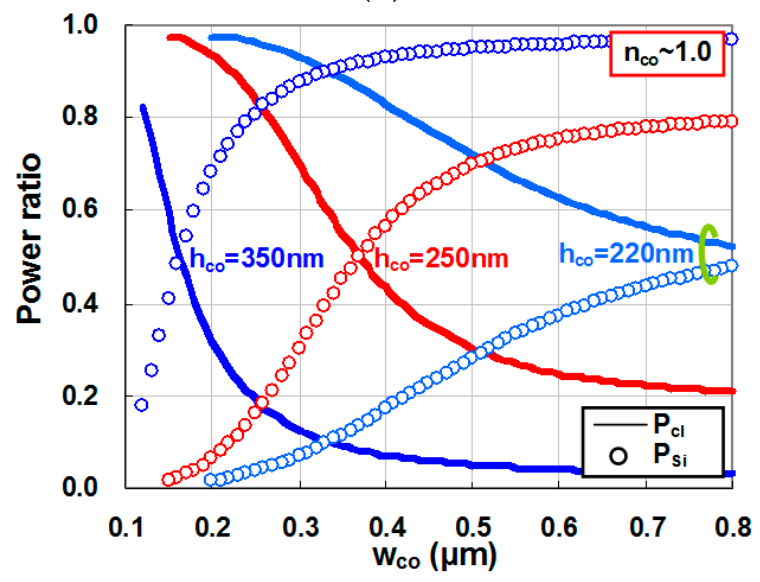

(d)

Figure 3. Cont. 


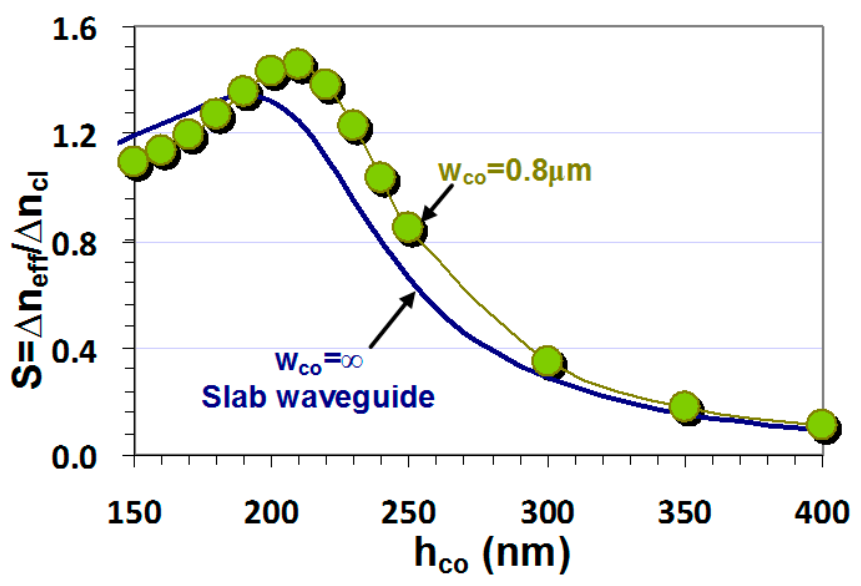

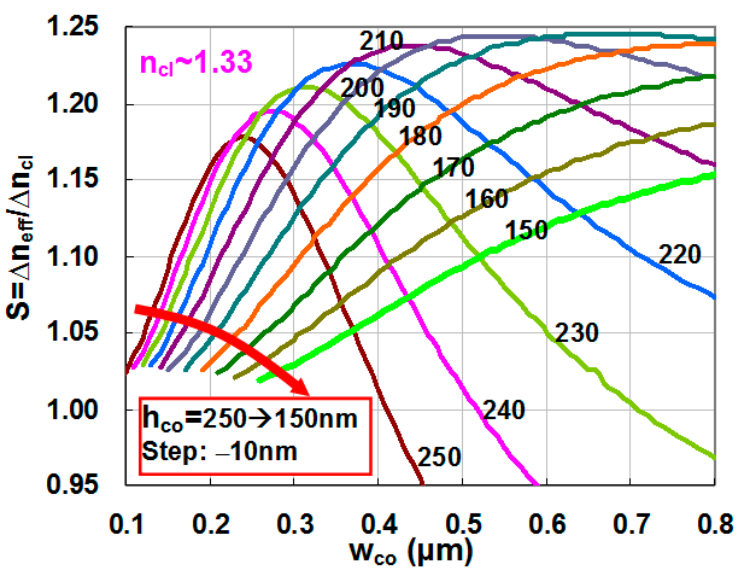

(f)

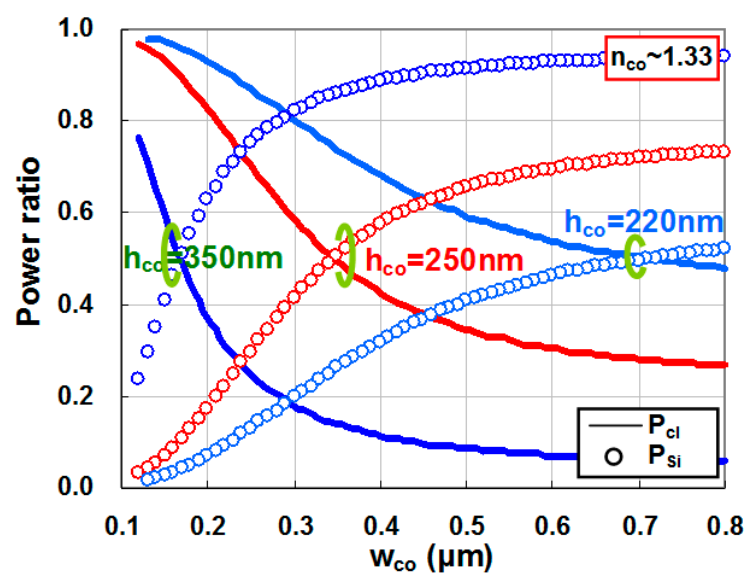

(g)

Figure 3. (a) The cross section of a suspended Si nanowire; (b) the calculated field distribution of the TM fundamental mode for a suspended Si nanowire waveguide with $\mathrm{h}_{\mathrm{co}}=220 \mathrm{~nm}, \mathrm{w}_{\mathrm{co}}=525 \mathrm{~nm}$, and $\mathrm{n}_{\mathrm{cl}}=1.0$; (c) the sensitivity of a suspended Si nanowire for gas sensing ( $\mathrm{ncl} \sim 1.0$ ); (d) the power confinement ratio in a suspended Si nanowire for gas sensing; (e) the calculated sensitivities for a suspended $\mathrm{Si}$ nanowire with $\mathrm{w}_{\mathrm{co}}=0.8 \mu \mathrm{m}$ and $\mathrm{w}_{\mathrm{co}}=\infty$ (slab waveguide); (f) the sensitivity of a suspended Si nanowire for liquid sensing $\left(\mathrm{n}_{\mathrm{cl}} \sim 1.33\right)$; and $(\mathrm{g})$ the power confinement ratio in a suspended Si nanowire for liquid sensing.

Figure $3 \mathrm{f}$ shows the sensitivity of a suspended $\mathrm{Si}$ nanowire for liquid sensing (i.e., $\mathrm{n}_{\mathrm{cl}} \sim 1.33$ ). Figure $3 g$ shows the power confinement ratios in the regions of core and cladding. It can be seen that the results are similar to those for gas sensing (i.e., $\mathrm{n}_{\mathrm{cl}} \sim 1.0$ ). The power ratio in the cladding region is higher because of the lower index-contrast. However, the maximal sensitivity is lower when there is a higher cladding index, as predicted in Equation (1).

\subsection{Nanofibers}

Nanofibers are another candidate for the use as a suspended waveguide for optical sensing. Since $\mathrm{Si}$ is considered for the core of the planar nanophotonic waveguides in the analysis above, we also choose nanofibers based on Si. Figure 4a shows the calculated waveguide sensitivity for a Si nanofiber with gas cladding as the core radius $\mathrm{R}$ varies from $0.06 \mu \mathrm{m}$ to $0.3 \mu \mathrm{m}$. The inset shows the cross section of the Si nanofiber. From this figure, one sees that there is an optimal core radius giving a maximal sensitivity, which is similar to the result for suspended Si nanowires. This could be explained as follows: when the core radius is small, the optical confinement is very weak and most power of the guided mode is in the cladding. In this case, the nanofiber behaves like in free space sensing. When the 
core radius becomes large, the optical confinement becomes very strong. Consequently the evanescent field is very small, which reduces the sensitivity. One might see it in Figure $4 \mathrm{a}$, when it is used for gas sensing, the optimal core radius is about $0.15 \mu \mathrm{m}$ and the maximal sensitivity $S_{\max }$ is about 1.43 , which is slightly lower than that of an optimized suspended Si nanowire (see Figure 3b) In contrast, for the case of liquid sensing, the optimal core radius is about $0.14 \mu \mathrm{m}$ and the maximal sensitivity $S_{\max }$ is about 1.2.

When one chooses the optimal core radius for the highest sensitivity, care must be taken because of the strong tolerance upon the core radius dimension. For example, when the core radius varies from the optimal value (i.e., $150 \mathrm{~nm}$ ) to $165 \mathrm{~nm}$, the sensitivity decreases from 1.43 to 0.943 and one has $\Delta S / \Delta \mathrm{R}=32.5 / \mu \mathrm{m}$ (here $\Delta \mathrm{R}$ is the radius variation and $\Delta S$ is the sensitivity decrease). This indicates that one should control the diameter of the nanofiber very carefully in order to achieve the maximal sensitivity. In contrast, the suspended Si nanowire is more tolerant to the lateral dimension variation $\Delta \mathrm{w}_{\mathrm{co}}$. For example, when the core width $\mathrm{w}_{\mathrm{co}}$ of a suspended waveguide (with $\mathrm{h}_{\mathrm{co}}=220 \mathrm{~nm}$ ) varies from the optimal value (i.e., $440 \mathrm{~nm}$ ) to $455 \mathrm{~nm}$, the sensitivity decreases from 1.477 to 1.475 and one has $\Delta S / \Delta \mathrm{w}_{\mathrm{CO}}=0.13 / \mu \mathrm{m}$, which is 240 times less than that of a silicon nanofiber. We note that it still not easy to fabricate Si nanofibers with the dimension s required for the highest sensitivity. On the other hand, it is well known that nanofibers have been fabricated successfully in the past years using $\mathrm{SiO}_{2}$ or polymer [23]. Figure $4 \mathrm{~b}$ shows the calculated sensitivity of a $\mathrm{SiO}_{2}$ nanofiber with air cladding. In this figure, it can be seen that one has a maximal sensitivity close to 1.0 when the core radius of the nanofiber is small. Due to the lower index contrast, one cannot obtain an enhanced waveguide sensitivity of more than 1.0 using $\mathrm{SiO}_{2}$ or polymer nanofibers.

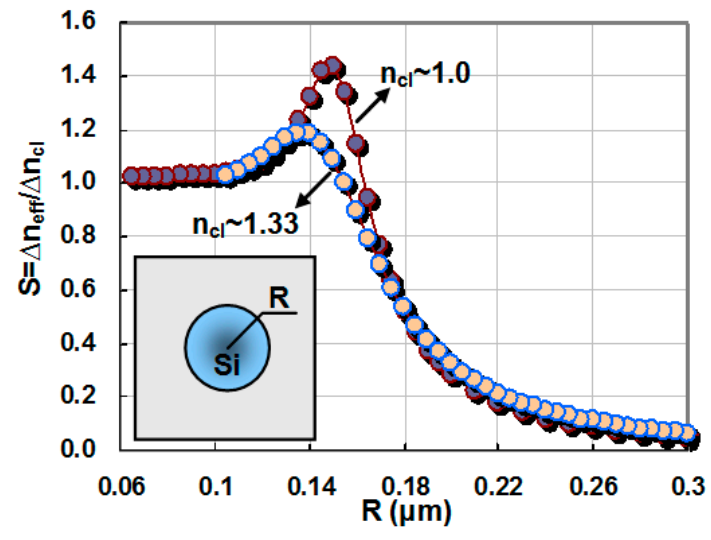

(a)

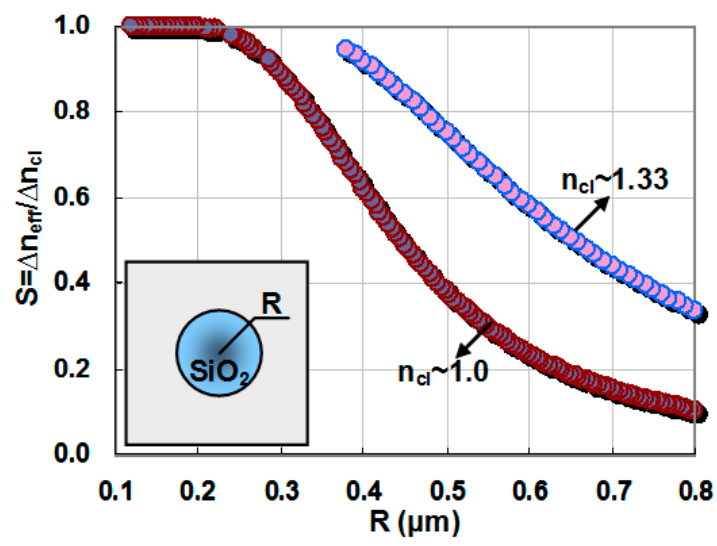

(b)

Figure 4. The sensitivity for a nanofiber with: (a) Si core; and (b) $\mathrm{SiO}_{2}$ core.

\section{Conclusions}

In this paper, a comparative study has been given for the sensitivity of several typical Si nanophotonic waveguides, including SOI nanowires, SOI nanoslot waveguides, suspended Si nanowires, and nanofibers. We have considered two cases of gas sensing $\left(\mathrm{n}_{\mathrm{cl}} \sim 1.0\right)$, and liquid sensing $\left(\mathrm{n}_{\mathrm{cl}} \sim 1.33\right)$. When using SOI nanowires (with a $\mathrm{SiO}_{2}$ buffer layer), the sensitivity for liquid sensing is higher than that for gas sensing, due to lower asymmetry in the vertical direction. Our calculations have also shown that it is possible to achieve a waveguide sensitivity higher than that of a free-space sensing ( $S=1.0)$ using SOI nanoslot waveguides, suspended Si nanowires, or Si nanofibers. Among them, the suspended Si nanowires give the highest sensitivity with an optimized core size. One should note that the fabrication process for suspended Si waveguides is more complex than that of standard SOI nanowires. Fortunately such a suspended waveguide can be achieved with supported arms by utilizing additional etching processes to remove the bottom-buffer layer [4,24,25]. When using an optimized suspended Si nanowire for gas sensing, the waveguide sensitivity could be as high as 
1.5, which is much higher than that of a SOI nanoslot waveguide. More importantly, the sensitivity of the optimal suspended Si nanowire decreases only very slightly, even when the core width has some variation (e.g., $\Delta \mathrm{w}= \pm 50 \mathrm{~nm}$ ). For an optimized Si nanofiber, the sensitivity could be as high as about 1.43 , which is close to that of an optimized suspended Si nanowire. However, the tolerance is very small (e.g., the deviation $< \pm 5 \mathrm{~nm}$ ) and one has to control the diameter of the nanofiber very carefully. The present comparative study has shown that suspended Si nanowire is a good choice to achieve ultra-high waveguide sensitivity.

Acknowledgments: This work was partially supported by the National Nature Science Foundation of China (61377023, 61422510, 91233208, and 6141101056), Zhejiang Provincial Natural Science Foundation of China (LY13F050002), The Doctoral Fund of Ministry of Education of China (No. 20120101110094) and the Program of Zhejiang Leading Team of Science and Technology Innovation (2010R50007).

Author Contributions: Y. Shi, K. Ma and D. Dai conceived and performed the simulations and the design of the devices reported in the paper. Y. Shi and D. Dai contributed to the writing and organization of the contents.

Conflicts of Interest: The authors declare no conflict of interest.

\section{References}

1. Densmore, A.; Xu, D.X.; Waldron, P.; Janz, S.; Cheben, P.; Lapointe, J.; Delâge, A.; Lamontagne, B.; Schmid, J.H.; Post, E. A silicon-on-insulator photonic wire based evanescent field sensor. IEEE Photonics Technol. Lett. 2006, 18, 2520-2522. [CrossRef]

2. Passaro, V.; Tullio, C.D.; Troia, B.; Notte, M.L.; Giannoccaro, G.; Leonardis, F.D. Recent advances in integrated photonic sensors. Sensors 2012, 12, 15558-15598. [CrossRef] [PubMed]

3. Wang, Q.; Farrell, G. All-fiber multimode-interference-based refractometer sensor: Proposal and design. Opt. Lett. 2006, 31, 317-319. [CrossRef] [PubMed]

4. Hu, J.; Dai, D. Cascaded-ring optical sensor with enhanced sensitivity by using suspended Si-nanowires. IEEE Photonics Technol. Lett. 2011, 23, 842-844.

5. De Leonardis, F.; Campanella, C.E.; Troia, B.; Perri, A.G.; Passaro, V. Performance of SOI Bragg Grating Ring Resonator for Nonlinear Sensing Applications. Sensors 2014, 14, 16017-16034. [CrossRef] [PubMed]

6. Armani, M.; Kulkarni, R.P.; Fraser, S.E.; Flagan, R.C.; Vahala, K.J. Label-free, single-molecule detection with optical microcavities. Science 2007, 317, 783-787. [CrossRef] [PubMed]

7. Zhou, Z.P.; Wu, H.M.; Feng, J.B.; Hou, J.; Yi, H.X.; Wang, X.J. Silicon nanophotonic devices based on resonance enhancement. J. Nanophotonics 2010, 4, 041001. [CrossRef]

8. Qing, D.-K.; Yamaguchi, I.; Itoh, K.; Murabayash, M. Coupling-wave structures for optical waveguide sensors. Opt. Rev. 1997, 4, 578-583. [CrossRef]

9. Troia, B.; Khokhar, A.Z.; Nedeljkovic, M.; Reynolds, S.A.; Hu, Y.; Mashanovich, G.Z.; Passaro, V.M.N. Design Procedure and Fabrication of Reproducible Silicon Vernier Devices for High-Performance Refractive Index Sensing. Sensors 2015, 15, 13548-13567. [CrossRef] [PubMed]

10. Wang, J.; Dai, D. Highly sensitive Si nanowire-based optical sensor using a Mach-Zehnder interferometer coupled microring. Opt. Lett. 2010, 35, 4229-4231. [CrossRef] [PubMed]

11. Chao, C.Y.; Guo, L.J. Design and optimization of microring resonators in biochemical sensing application. J. Lightwave Technol. 2006, 24, 1395-1402. [CrossRef]

12. Dai, D. Highly sensitive digital optical sensor based on cascaded high-Q ring-resonators. Opt. Express 2009, 17, 23817-23822. [CrossRef] [PubMed]

13. Claes, T.; Bogaerts, W.; Bienstman, P. Experimental characterization of a silicon photonic biosensor consisting of two cascaded ring resonators based on the Vernier-effect and introduction of a curve fitting method for an improved detection limit. Opt. Express 2010, 18, 22747-22761. [CrossRef] [PubMed]

14. Jin, L.; Li, M.; He, J.-J. Optical waveguide double-ring sensor using intensity interrogation with a low-cost broadband source. Opt. Lett. 2011, 36, 1128-1130.

15. Veldhuis, G.J.; Parriaux, O.; Hoekstra, H.J.W.M.; Lambeck, P.V. Sensitivity enhancement in evanescent optical waveguide sensors. J. Lightwave Technol. 2000, 18, 677-682. [CrossRef]

16. Fink, Y.; Ripin, D.J.; Fan, S.; Chen, C.; Joannopoulos, J.D.; Thomas, E.L. Guiding optical light in air using an all-dielectric structure. J. Lightwave Technol. 1999, 17, 2039-2041. [CrossRef] 
17. Almeida, V.R.; Xu, Q.; Barrios, C.A.; Lipson, M. Guiding and confining light in void nanostructure. Opt. Lett. 2004, 29, 1209-1211. [CrossRef] [PubMed]

18. Gylfason, K.B.; Carlborg, C.F.; Kazmierczak, A.; Dortu, F.; Sohlström, H.; Vivien, L.; Barrios, C.A.; Wijngaart, W.; Stemme, G. On-chip temperature compensation in an integrated slot-waveguide ring resonator refractive index sensor array. Opt. Express 2010, 18, 3226-3237. [CrossRef] [PubMed]

19. Dell'Olio, F.; Passaro, V.M. Optical sensing by optimized silicon slot waveguides. Opt. Express 2007, 15, 4977-4993. [CrossRef] [PubMed]

20. Kargar, A.; Chao, C.-Y. Design and optimization of waveguide sensitivity in slot microring sensors. J. Opt. Soc. Am. A 2011, 28, 596-603. [CrossRef] [PubMed]

21. Stievater, T.H.; Park, D.; Rabinovich, W.S.; Pruessner, M.W.; Kanakaraju, S.; Richardson, C.J.; Khurgin, J.B. Enhanced electro-optic phase shifts in suspended waveguides. Opt. Express 2010, 18, 885-892. [CrossRef] [PubMed]

22. Yang, B.; Sheng, Z.; Dai, D. Design of Suspended SU-8 Optical Waveguides for Ultrasmall Bendings. In Proceedings of the 2009 Asia Communications and Photonics Conference and Exhibition (ACP), Shanghai, China, 2-6 November 2009.

23. Tong, L.; Gattass, R.R.; Ashcom, J.B.; He, S.; Lou, J.; Shen, M.; Maxwell, I.; Mazur, E. Subwavelength-diameter silica wires for low-loss optical wave guiding. Nature 2003, 426, 816-819. [CrossRef] [PubMed]

24. Martinez, L.; Lipson, M. High confinement suspended micro-ring resonators in silicon-on-insulator. Opt. Express 2006, 13, 6259-6263. [CrossRef]

25. Wang, X.; Guan, X.; Huang, Q.; Zheng, J.; Shi, Y.; Dai, D. Suspended Submicron-disk Resonator on Silicon for Optical Sensing. Opt. Lett. 2013, 38, 5405-5408. [CrossRef] [PubMed]

(C) 2016 by the authors; licensee MDPI, Basel, Switzerland. This article is an open access article distributed under the terms and conditions of the Creative Commons by Attribution (CC-BY) license (http:/ / creativecommons.org/licenses/by/4.0/). 